\title{
Long Term Evolution (LTE)
}

\author{
Emad Kazi ${ }^{1}$, Rajan Pillai ${ }^{2}$, Uzair Qureshi ${ }^{3}$, Awab Fakih ${ }^{4}$ \\ 1,2,3,4 (Electronics and Telecommunication, Anjuman-I-Islam's Kalsekar technical campus (AIKTC), Mumbai \\ University, India)
}

\begin{abstract}
The number of people using mobile phone in the world has exceeded 4.5 billion and this figure is continuing to grow. For the past several years, mobile data traffic such as internet access, the downloading of music and video communication has been nearly tripling every year. With the popularity of smartphones, mobile data traffic will increase 200 times in the 7 to 8 years upto 2020.There are high expectations that Long Term Evolution (LTE) which is known as $3.9 \mathrm{G}$ wireless system will be a new service platform that can support a huge amount of mobile data traffic. This paper describes the features, technology and network architecture of LTE \& also provides an overview of next generation telecommunication network LTE, which is started commercially in December 2010 in Japan (started by DOCOMO), realizing high speed wireless access. It also outlines the further trends towards a further speed increase.
\end{abstract}

Keywords-Circuit Switching, GSM, HSPA, LTE, Packet Switching, WiMAX

\section{Introduction}

In times when mobile devices are getting more popular the mobile network are becoming more and more important too. Websites are not same they used to be 10 years ago. They consist of with quality pictures, animation, flash application and more. Also the demand for mobile internet grew significantly. According to Opera's Web browser the number of pages viewed has risen from about 1.8 billion pages in January 2008 to 23 billion in January 2010.

All these leading to an increase amount of data. Even calling and messaging with the mobile phone requires more data today. There are not only simple calls and short messaging anymore, there is also video telephony, multimedia messaging and emailing possible nowadays. Also netbooks and tablet pcs are becoming more popular than ever. People want to access the internet with their phones and notebooks from everywhere.

Services like the Internet Protocol television (IPTV) also highly increased the need for higher transfer rates and better availability of mobile internet connection grows more and more. So it is just a matter of time until current telecommunication networks reach their limit.

The number of people using mobile phone in the world has exceeded 4.5 billion and this figure is continuing to grow. For the past several years, mobile data traffic such as internet access, the downloading of music and video communication has been nearly tripling every year. With the popularity of smartphones, mobile data traffic will increase 200 times in the 7 to 8 years upto 2020 .

The next generation networks Worldwide Interoperability for Microwave Access (WiMAX) and LongTerm Evolution LTE) promise to bring better transfer rates, lower latency, better availability and more to fulfill the needs of the customers, but they also have to be more effective.

They should not be too expensive for providers, they should reach more devices with less costs. Otherwise they would be unprofitable for the carriers or it would take too much time until the new communication networks would cover a large area. And maybe until then other communication networks would be developed.

That's what happened to the 3rd generation telecommunication networks. They are still not fully available everywhere and newer generation networks such as WiMAX and LTE are on the way or even already out. Will LTE and WiMAX be the next generation telecommunication networks?

\section{Overview}

Currently the mobile network infrastructure comprises overlay networks including $2 \mathrm{G}$ and $3 \mathrm{G}$ technologies.

Global System for Mobile Communications (GSM) is a 2nd generation mobile network and provides circuit-switched communication. It was enhanced by General Packet Radio Service (GPRS), also known as $2.5 \mathrm{G}$, and Enhanced Data rates for GSM Evolution (EDGE), also known as $2.75 \mathrm{G}$, providing IP functionality and data transfer rates up to $1.3 \mathrm{Mbps}$ in the downlink and $653 \mathrm{kbps}$ in the uplink.

On the side of the 3rd generation communication networks, there is Universal Mobile Telecommunications System (UMTS) and its enhancements High-Speed Downlink Packet Access (HSDPA) and High-Speed Uplink Packet Access (HSUPA), both also known as 3.5G networks. Maximum transfer rates 
by 3rd generation mobile networks can be reached with High-speed Data Access (HSPA) Evolution. 28 Mbps in the downlink and $5.76 \mathrm{Mbps}$ in the uplink are possible.

All these technologies have been standardized by either the 3rd Generation Partnership Project (3GPP) or the Institute of Electrical and Electronic Engineers (IEEE). 3rd generation mobile networks can reach quite high transfer rates compared to 2nd generation networks but they have higher service costs. Compared to GSM, UMTS and HSDPA do not provide full coverage.

In order to form new communication standards regarding $4 \mathrm{G}$, the International Telecommunication Union (ITU) launched the International Mobile Telecommunications (IMT)-Advanced initiative. WiMAX and Long-Term Evolution (LTE) meets most of the requirements of IMT-Advanced, however they are just considered as $3.9 \mathrm{G}$ even though telecommunication companies use the term $4 \mathrm{G}$ when marketing WiMAX and LTE.

LTE commonly referred to as $4 \mathrm{G}$ or next generation technology wireless communication is the new standard for nationwide public safety broadband. LTE is the avenue (road) for fully bringing public safety fully into the digital age. This network will foster further development of application customized for public safety and help make first responders operation more effective and efficient. Unlike the current wireless environment where interoperability among public safety devices and across jurisdiction is deficient, the nationwide network Interoperability

LTE is the standard that is supported by public safety and has been adopted by the Federal Communication (FCC) as the technology platform for public safety broadband in the $700 \mathrm{MHz}$ band.

Simply stated, LTE network are much faster than previous network used within public safety with speed upto 10 times much faster than $3 \mathrm{G}$ network.

\section{History}

Public safety Land Mobile Radio (LMR) use began with voice only radio back in early 1940's. Data transmissions were possible over LMR system but limited to simple text based application. Large data file, photograph, videos, etc. could not be viewed on early mobile data computers because data speeds on these networks were generally limited to 19.2 kbps.

Let's examine the LTE standard and related technology, and discuss how this new technology can enhance public safety responders to emergencies.LTE was first used in the commercially broadband world with all the major wireless carriers adopting the standard. Due to which today, speed and data transfer capabilities we would have expected at our desktop a few short years ago are available to the public safety responder in the field.

\section{AboutLTE}

Long Term Evolution also known as LTE was developed by the 3rd Generation Partnership Project (3GPP), collaboration between groups of telecommunication associations. It was released in the $4^{\text {th }}$ quarter of 2008. The 3GPP partner from the US is the Alliance for Telecommunications Industry Solutions which members include telecommunication companies, such as AT\&T, Cisco and Verizon. The LTE standard is officially known as "document 3GPP Release 8". LTE Release 8 is sometimes also called as $3.9 \mathrm{G}$ because it almost achieves full compliance with IMT-Advanced.

Figure 1 shows trends of wireless access systems. High-speed wireless access systems that accommodate mobile data communications traffic can be roughly classified into two trends: mobile phone-based and wireless LAN-based. LTE is an enhanced standard of High Speed Packet Access (HSPA), which is a highspeed data communications standard for Wideband Code Division Multiple Access (W-CDMA), a 3rd generation mobile telecommunications $(3 \mathrm{G})$ system. To realize high-speed communications with a downlink of at least $100 \mathrm{Mb} / \mathrm{s}$ and uplink of at least $50 \mathrm{Mb} / \mathrm{s}$, LTE was specified as Release 8 in March 2009 by the 3rd Generation Partnership Project (3GPP), the W-CDMA standardization organization. Smooth transition to the $4^{\text {th }}$ generation $(4 \mathrm{G})$ is intended by providing services using the technologies developed for $4 \mathrm{G}$ and the same frequency bands as for 3G. While HSPA is called the 3.5th generation (3.5G), LTE is referred to as the 3.9th generation $(3.9 \mathrm{G})$ in the sense that the system is extremely close to $4 \mathrm{G}$.

The requirements for LTE have been discussed by the 3GPP starting in March 2005. Up to 3G, both the Circuit Switched (CS) domain for voice communications and Packet Switched (PS) domain for data communications were supported. With LTE, however, in order to reduce costs by simplifying the system, focus is placed on data communications, which is expected to be the mainstream of traffic inthe future, to support the PS domain only. Voice services are assumed to use voice over IP (VoIP) for provision in the PS domain as data communications. Another aim is to significantly improve delay time, which has been an issue with $3 \mathrm{G}$. 


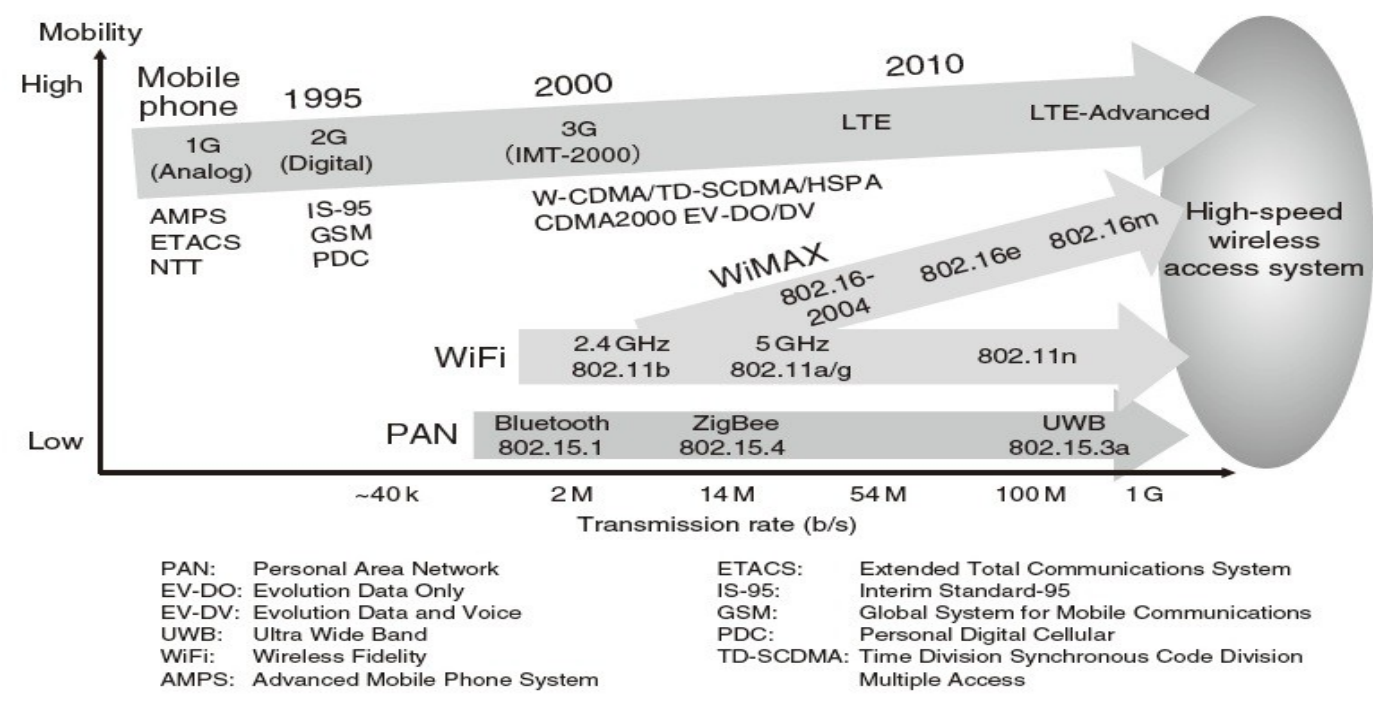

Fig1: trends of high-speed wireless access mobile communication system

The requirements of LTE finally approved by the 3GPP as TR25.9132) can be summarized as follows:

1) Specialization in data communications

(Packet switching)

2) Support for a variable bandwidth (1.4 to $20 \mathrm{MHz})$

3) Realization of low latency

i. Connection delay: up to $100 \mathrm{~ms}$

ii. Transfer delay: up to $5 \mathrm{~ms}$ (wireless sections)

4) Realization of high speed

i. Downlink: at least $100 \mathrm{Mb} / \mathrm{s}$

ii. Uplink: at least $50 \mathrm{Mb} / \mathrm{s}$

5) Improvement of frequency utilization efficiency (with reference to $3.5 \mathrm{G}$ )

i. Downlink: at least triple

ii. Uplink: at least double

6) Coexistence with the existing systems

$(3 \mathrm{G}$ and $3.5 \mathrm{G})$

The following sections outline the key technologies and network architecture adopted for LTE to satisfy the requirements listed above.

\section{Key Technologies Used}

The biggest purpose of LTE is the realization of high-speed wireless access. For higher speed, it is necessary to improve utilization efficiency of the available frequency bandwidth and expand the bandwidth. For that reason, LTE uses the following technologies:

1) Wireless access system

The downlink uses orthogonal frequency division multiple access (OFDMA), which is also applied to wireless LAN and other systems. OFDMA assigns channels (subcarriers) to users along the frequency and time axes. The frequency utilization efficiency can be improved by assigning channels with higher transmission efficiency according to the wireless access environment of the user. It also features high resistance to multipath interference. As compared with the code division multiple access (CDMA) system used for $3 \mathrm{G}$ and $3.5 \mathrm{G}$, the same frequency width can accommodate three to four times as much data. On the other hand, the uplink uses single-carrier (SC) FDMA, which has a small peak-to-average power ratio of transmission signals and allows easy high output transmission. 
2) Application of multi-antenna technology

Support for transmitting and receiving technology that uses multiple antennas both at the base station and terminal has allowed an improvement in the frequency utilization efficiency and expansion of coverage. The most suitable technique can be selected according to factors including the propagation environment out of options such as multiple input multiple output (MIMO), transmit diversity and beam forming. MIMO is a technology to transmit and receive different data on the same frequency by using multiple antennas. The frequency utilization efficiency can be improved according to the number of antennas. LTE supports up to fourstream MIMO access for downlink in terms of the specifications.

\section{3) Expansion of bandwidth}

While 3.5G HSPA uses a frequency band with a width of $5 \mathrm{MHz}$, LTE supports up to $20 \mathrm{MHz}$ bandwidth. Bandwidth and transmission rate are nearly proportional to each other and a quadrupled bandwidth allows a speed increase of four times. In addition, LTE supports 1.4, 3, 5, 10, 15 and $20 \mathrm{MHz}$ bandwidths to provide applicability to frequencies of different bandwidths.

4) Reduction of transmission delay

To support VoIP and allow comfortable use of real-time applications such as online games, the transmission delay must be minimized. LTE has achieved this by adopting a wireless channel structure exclusively for short wireless frame lengths and packet transmission. Regarding the wireless network architecture, the two- layer structure with wireless base stations and a wireless control station, which was used for generations up to $3.5 \mathrm{G}$, has been revised to provide a flat structure without the wireless control station, as shown in Figure 2. In this way, one-way transmission delay of $5 \mathrm{~ms}$ maximum in the optimum condition without congestion has been realized.

Table 1 shows a comparison of the specifications and performance of LTE and $3 \mathrm{G}$ and $3.5 \mathrm{G}$ wireless systems.

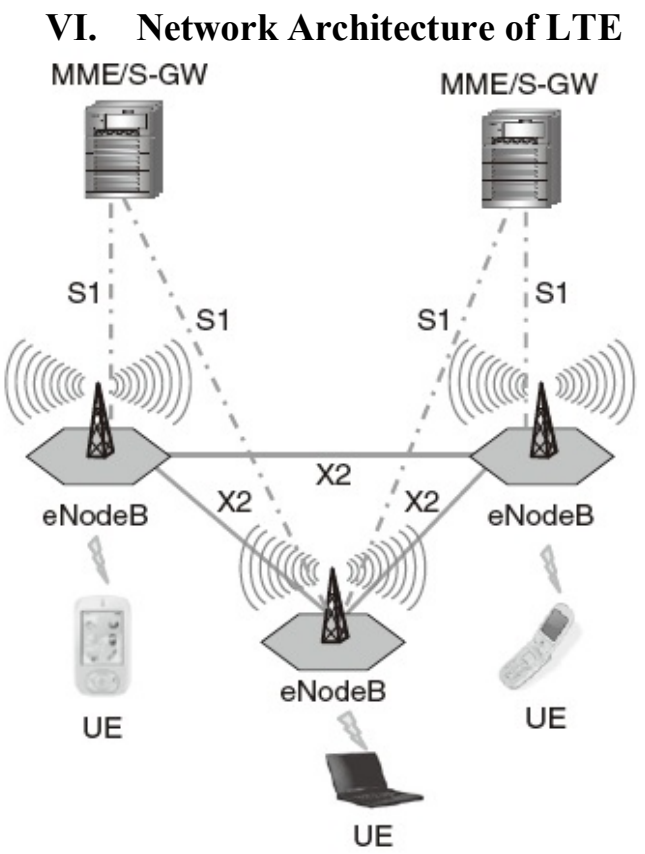

Fig 2: LTE wireless architecture

The discussion on the core network for accommodating LTE started under the name of system architecture evolution (SAE) by 3GPP. As a result, Evolved Packet Core (EPC) as a next-generation IP-based core network was established as the standard specification of 3GPP Release 8 as with LTE. EPC is architecture exclusively for data communications and has the following characteristics.

1) Packet-based architecture

Data communications including Internet access is expected to be the mainstream of traffic in the future and only packet switching is specified to achieve more simplified and efficient networks. For circuit switching services provided in $3 \mathrm{G}$ systems, capabilities of IP multimedia subsystem (IMS) are used for offering equivalent services. 
Table 1: Comparission of high-speed wireless access system

\begin{tabular}{|c|c|c|c|c|c|}
\hline & \multirow{2}{*}{$\begin{array}{c}\text { W-CDMA } \\
(3 G)\end{array}$} & \multirow{2}{*}{$\begin{array}{c}\text { HSDPAEUL } \\
(3.5 \mathrm{G})\end{array}$} & \multicolumn{2}{|c|}{ CDMA2000 1xEV-DO } & \multirow{2}{*}{ LTE } \\
\hline & & & Rev.0 & Rev.A & \\
\hline Multiplexing scheme & $\begin{array}{l}\text { DL: CDMA } \\
\text { UL: CDMA }\end{array}$ & $\begin{array}{l}\text { DL: CDMA } \\
\text { UL: CDMA }\end{array}$ & $\begin{array}{l}\text { DL: CDMA } \\
\text { UL: CDMA }\end{array}$ & $\begin{array}{l}\text { DL: CDMA } \\
\text { UL: CDMA }\end{array}$ & $\begin{array}{l}\text { DL: OFDMA } \\
\text { UL: SC-FDMA }\end{array}$ \\
\hline Frequency band & $5 \mathrm{MHz}$ & $5 \mathrm{MHz}$ & $1.25 \mathrm{MHz}$ & $1.25 \mathrm{MHz}$ & $20 \mathrm{MHz}$ \\
\hline Modulation scheme & HPSK, QPSK & $\begin{array}{l}\text { HPSK, QPSK } \\
\text { 16QAM }\end{array}$ & $\begin{array}{l}\text { BPSK, QPSK } \\
\text { 8PSK, 16QAM } \\
\end{array}$ & $\begin{array}{l}\text { BPSK, QPSK } \\
\text { 8PSK, 16QAM } \\
\end{array}$ & $\begin{array}{l}\text { QPSK, 16QAM } \\
\text { 64QAM, etc. }\end{array}$ \\
\hline $\begin{array}{l}\text { Data rate } \\
\text { (Maximum) }\end{array}$ & $\begin{array}{l}\text { DL: } 384 \mathrm{~kb} / \mathrm{s} \\
\text { UL: } 64 \mathrm{~kb} / \mathrm{s}\end{array}$ & $\begin{array}{l}\mathrm{DL}: 14.4 \mathrm{Mb} / \mathrm{s} \\
\mathrm{UL}: 5.7 \mathrm{Mb} / \mathrm{s}\end{array}$ & $\begin{array}{l}\mathrm{DL}: 2.4 \mathrm{Mb} / \mathrm{s} \\
\mathrm{UL}: 154 \mathrm{~kb} / \mathrm{s}\end{array}$ & $\begin{array}{l}\mathrm{DL}: 3.1 \mathrm{Mb} / \mathrm{s} \\
\text { ULا } 1.8 \mathrm{Mb} / \mathrm{s}\end{array}$ & $\begin{array}{l}\mathrm{DL}: 325 \mathrm{Mb} / \mathrm{s} \\
\mathrm{UL:} 86 \mathrm{Mb} / \mathrm{s}\end{array}$ \\
\hline $\begin{array}{l}\text { Start of commercial } \\
\text { service }\end{array}$ & 2000 & $\begin{array}{l}\text { HSDPA: } 2006 \\
\text { EUL: } \quad 2008 \\
\end{array}$ & 2003 & 2006 & 2009 \\
\hline
\end{tabular}

2) Always-on connection

To reduce connection delay, EPC establishes a logical transmission path when the terminal isturned on and registered with the network. On a core network, the established status of the transmission path is always maintained, and only wireless connection settings between the mobile terminal and base station need to be configured for actual communications.In this way, the connection delay can be significantly reduced.This, together with the simplification of protocols in the wireless sections and the flattening of the wireless network architecture mentioned above, has achieved a connection delay time of $100 \mathrm{~ms}$ maximum.

3) Accommodation of different wireless system

EPC has been specified with the focus on accommodating LTE. However, it is established as a common core network capable of not only accommodating various wireless access systems such as CDMA2000, WiMAX and Wi-Fi in addition to 3GPP-compliant wireless systems including 3G W-CDMA and 3.5G HSPA but also handover between these different wireless access systems. Network architecture independent of the wireless access systems has been constructed by widely incorporating general-purpose IPs.

An overview of the EPC network architecture is shown in Figure 3. A Mobility Management Entity (MME) manages the authentication and movement of terminals. It also handles interworking with the existing W-CDMA and HSPA and configures user data transfer paths in the EPC. A Serving Gateway (S-GW) accommodates enhanced Node Bs (eNodeBs), which are LTE wireless base stations, and transfers user data. It is also provided with a function of an anchor point that switches user data between LTE and the existing WCDMA/ HSPA. A Packet data network Gateway (P-GW) has a function to connect with IP service systems operated by operators themselves such as IMS and IP service providers other than operators and assigns IP addresses to mobile terminals.

It also accommodates non-3GPP wireless access systems such as WiMAX and Wi-Fi. Policy and Charging Rules Function (PCRF) establishes policies including QoS and charging rules in the EPC. In the PGW and S-GW, policy control and charging control take place according to the instructions from PCRF.

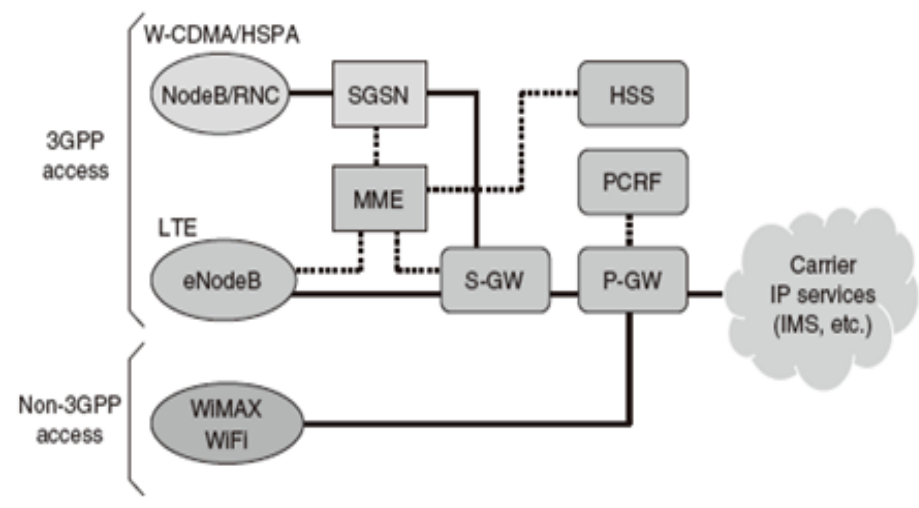

Fig 3: EPC Network Architecture 
A home subscriber server (HSS) stores user profiles such as various identifiers for identifying users and information about services to which users subscribe. When a user accesses the network via an eNodeB or NodeB, MME references the profile stored in the HSS for user authentication and service authentication.

A serving GPRS support node (SGSN) handles the authentication and location management of terminals connected to W-CDMA and HSPA. General-purpose IPs is used to connect between these nodes, which has enabled EPC to realize efficient transfer of high-speed data communications independent of the wireless access systems.

\section{Conclusion}

This paper has described LTE, whichrealizes high-speed wireless access services, byoutlining its characteristics, key technologiesand network architecture. For even higher-speed wireless access, 3GPP is already workingon the standardization of LTE-Advanced. LTE-Advanced is a wireless access system positioned as $4 \mathrm{G}$ and based on the LTE technology, as its nameindicates. It is targeted to realize high-speed transmission of $1 \mathrm{~GB} / \mathrm{s}$.

This can be achievedby using carrier aggregation technology, whichaggregates multiple wireless bands of $20 \mathrm{MHzin} \mathrm{LTE} \mathrm{for} \mathrm{a} \mathrm{high} \mathrm{bandwidth} \mathrm{of} \mathrm{up} \mathrm{to} 100 \mathrm{MHz}$, and enhancing MIMO to support up to eightstreams. Fujitsu has already been developingLTE terminals, base stations and core networkdevices and various elemental technologiesfor realizing these devices. Fujitsu is alsoworking on the development of management andengineering technologies required for efficientlybuilding and operating LTE and EPC and hasbeen contributing to the start of commercial LTEservices. Fujitsu intends to continue to work ondevelopment for the full-scale diffusion of LTE. In addition, for the realization of LTE-Advanced, Fujitsu will move ahead with unceasing research and development from the standardization activities to commercialization.

Currently, the key areas these R\&D engineers are looking at are things like traffic and signaling load patterns: developing models and feedback methodologies for network testing and optimization.

Another key area is to look at changing user behavior, and the effect on the network, as new smartphones and other devices are introduced. The goal is to complement high quality lab-based work with reallife fieldwork to arrive at the smartest possible solution. We believe this kind of teamwork is vital not only to gain a deep technical understanding of LTE networks, but also of how different customers use features in different ways. Getting this end-to-end and top to bottom view of how LTE works in the field - and continuously feeding this information back into the design, implementation and testing process - has helped create the highest-performing and most stable LTE platforms on the market.

Thus we conclude that Long Term Evolution (LTE) is now clearly the technology of choice for Next Generation Technology, a standard developed by the 3GPP. Early 3GPP technologies included GSM, EDGE, UMTS and HSPA, all globally successful technologies based on FDD spectrum allocations, using a separate channel for uplink and downlink. This approach of using separate channels for subscriber information has been often used and is well understood. The standardized TDD flavor for LTE, conceived simultaneously with the FDD version, is now at a point where it is attracting global attention and providing new possibilities.

LTE still faces some deployment challenges (which are currently being studied).

\section{ACKNOWLEDGMENT}

We express sincere gratitude to our guide Asst. Professor Mr. Awab Fakih for his support and valuable guidance. He has motivated us throughout the course of the paper to work harder and achieve set goals. We are also highly grateful toAsst. Professor Mr. Mujeeb Tamboli for providing us this wonderful topic .We would also be highly grateful to Asst. ProfessorMrs. Chaya S Ravi and Asst. ProfessorMr. Afzal Shaikh for helping us. We are also highly grateful to Asst. ProfessorMr. Ramjan Khatik, Head of Department (EXTC) and the Director, Mr. Razzak Honnutagi for providing the facilities and conductive environment.

Authors gratefully acknowledge the financial support by their parent $\&$ family members in the present work. Special thanks to our family and friends to encourage us and provide us with practical suggestions for the improvement of our paper. 


\section{References}

[1] R.Nossenson, "Long-term evolution network architecture," in Microwaves, Communications, Antennas and Electronics Systems, 2009, COMCAS 2009, IEEE International Conference, 2009

[2] Tsuguo Kato,Next-Generation Mobile Network, FUJITSU Sci. Tech. J., Vol. 48, No. 1, January 2012.

[3] Dieter Eberle, "LTE vs. WiMAX: 4th generation telecommunication networks", Computer Engineering B.Sc. ,Berlin Institute of Technology, Germany

[2] "Long term evolution (LTE): A technical overview", Motorola, Inc., Schaumburg, IL http://business.motorola.com/experiencelte/pdf/LTE TechnicalOverview.pdf.

[4] "LTE: a global success story", (C) Ericsson AB 2012

[5] Ahmed Hamza, "Long Term Evolution (LTE)", Network Systems Laboratory, Simon Fraser University, Oct2009

[7] LTE Network Architecture. http://www.tutorialspoint.com/lte/lte network architecture.htm 